\title{
Monovinyl Sulfone $\beta$-Cyclodextrin. A Flexible Drug Carrier System
}

\author{
Teresa del Castillo, ${ }^{[c]}$ Julia Marales-Sanfrutos, ${ }^{[a, b]}$ Francisco Santoyo-González, ${ }^{[\mathrm{a}]}$ \\ Stefan Magez, ${ }^{[d]}$ F. Javier Lopez-Jaramillo, ${ }^{*[a]}$ and Jose A. Garcia-Salcedo ${ }^{[c]}$
}

\begin{abstract}
Cyclodextrins have been conjugated to target various receptors and have also been functionalized with carbohydrates for targeting specific organs. However, this approach is based on a rigid design that implies the ad hoc synthesis of each cyclodextrin-targeting agent conjugate. We hypothesized that: 1) a modular design that decouples the carrier function from the targeting function leads to a flexible system, 2) combining the reactivity of the vinyl sulfone group toward biomolecules that act as targeting agents with the ability of cyclodextrin to form complexes with a wide range of drugs may yield a versatile system that allows the targeting of different organs with differ-
\end{abstract}

ent drugs, and 3) the higher reactivity of histidine residues toward the vinyl sulfone group can be exploited to couple the cyclodextrin to the targeting system with a degree of regioselectivity. As a proof of concept, we synthesized a monovinyl sulfone $\beta$-cyclodextrin (module responsible for the payload), which, after coupling to recombinant antibody fragments raised against Trypanosoma brucei (module responsible for targeting) and loading with nitrofurazone (module responsible for therapeutic action) resulted in an effective delivery system that targets the surface of the parasites and shows trypanocidal activity.

\section{Introduction}

Ever since Paul Ehrlich coined the magic bullet concept (i.e., drugs that go straight to their intended cell structure target while leaving healthy tissues unaffected), much effort has been devoted to the delivery of drugs to specific physiological sites where pharmacological activity is required, particularly in the field of cancer therapy. ${ }^{[1]}$ The strategies for drug targeting are frequently divided into passive targeting and active targeting. ${ }^{[2]}$ Passive targeting is based on the physical and chemical properties of the carrier and the differences in the biochemical and physiological characteristics of the target tissue (i.e., en-

[a] Dr. J. Marales-Sanfrutos, ${ }^{+}$Prof. F. Santoyo-González, Dr. F. J. Lopez-Jaramillo Departamento de Química Orgánica, Instituto de Biotecnología Universidad de Granada, 18071 Granada (Spain) E-mail: fjljara@ugr.es

[b] Dr. J. Marales-Sanfrutos

Current address: Department of Chemistry Chemistry Research Laboratory University of Oxford, OX1 3TA Oxford (UK)

[c] Dr. T. del Castillo, ${ }^{+}$Dr. J. A. Garcia-Salcedo Hospital Universitario San Cecilio

Instituto de Investigaciones Biosanitarias de Granada FIBAO, Granada (Spain)

and

Instituto de Parasitología y Biomedicina López Neyra CSIC, Granada (Spain)

[d] Dr. S. Magez

Laboratory for Cellular and Molecular Immunology

Vrije Universiteit Brussel, Brussels (Belgium)

and

Department of Molecular and Cellular Interactions

VIB, Brussels (Belgium)

$\left.{ }^{[+}\right]$These authors contributed equally to this work.

Supporting information for this article is available on the WWW under http://dx.doi.org/10.1002/cmdc.201300385. hanced permeability and retention (EPR) effect ${ }^{[3]}$ to achieve a high target/non-target ratio at the site of action. However, the random nature of this approach makes it difficult to control the process, and active targeting is an appealing alternative. Active targeting relies on the use of specific interactions to promote not only the specificity, but also to increase the local concentration of the therapeutic agent at the site of action and, in some cases, to provoke its internalization mediated by receptors. ${ }^{[4]}$

Given the inherent nanometer-scale functions of the biological components of living cells, nanoscale materials are promising in medicine. Size is relevant in the context of drug delivery, and carriers larger than $400 \mathrm{~nm}$ in diameter are captured by the reticuloendothelial system. ${ }^{[5]}$ Various nanoscale materials have been described as carriers, and can be grouped into five general categories: 1) nanospheres, 2) liposomes, 3) emulsions, 4) polymeric micelles, and 5) water-soluble polymeric carriers including both naturally occurring polymers (i.e., proteins and carbohydrates) and synthetic polymers. ${ }^{[2,4 a, 6]}$

In active targeting, interactions with the specific receptor are mediated by biomolecules that act as targeting agents. The use of antibodies in their native state as targeting agents, either as fragments or as antibody mimics, is well established. ${ }^{[7]}$ In oncology the fact that cancer cells overexpress receptors to maintain their high metabolic rates has also been exploited in folate targeting ${ }^{[8]}$ and in transferrin-mediated targeting. ${ }^{[9]}$ However, regardless the targeting agent and the carrier, the coupling of the elements comprising the drug delivery system is an important issue. Methods of protein chemistry have been successfully implemented for the direct conjugation of drugs to antibodies. ${ }^{[10]}$ For the particular case of immunotoxins, the 
use of recombinant DNA techniques is a feasible approach to obtain toxin-antibody systems as fusion proteins. ${ }^{[7 b, 10]}$ In general, nanoscale drug delivery systems have been conjugated to targeting agents by classical chemical functionalization methods, ${ }^{[7 a, 9 a, 11,12]}$ and by avidin-biotin affinity. ${ }^{[13]}$ Another important issue is the stability of the linkage, which may explain the failure of first-generation conjugates that led to premature release of the drugs in the blood, or to no release at the site of action. Second-generation linkers were designed to be cleaved within intracellular organelles through decreased $\mathrm{pH}$ (acid-labile hydrazone $)^{[7 \mathrm{~d}]}$ or a reductive environment. ${ }^{[14]}$

Cyclodextrins (CDs) have been identified as promising nanoscale carriers. ${ }^{[15]}$ They are cyclic oligosaccharides composed of six, seven, or eight D-glucopyranose units referred to as $\alpha-, \beta$-, and $\gamma$-cyclodextrin, respectively, linked by $\alpha(1 \rightarrow 4)$ bonds enclosing a lipophilic central cavity. They are well known for their capacity to form inclusion complexes with a wide variety of drugs. ${ }^{[16]}$ In the context of active drug delivery, CDs have been conveniently conjugated to target estrogen receptors, folate receptors, transferrin receptors, and integrins, among others, as well as functionalized with carbohydrates for targeting different organs. ${ }^{[15]}$ This approach is based on the ad hoc synthesis of each cyclodextrin-targeting agent conjugate and leads to a rigid design that constrains the use of cyclodextrin in active drug delivery.

We hypothesized that a modular design that decouples the carrier function from the targeting function may lead to a flexible system. As a proof of concept, herein we report the synthesis of a monovinyl sulfone functionalized $\beta$-cyclodextrin (VSCD) that combines the capacity of CD to carry different drugs with the good reactivity of the vinyl sulfone group toward the biomolecule, acting as targeting agent. Thus, the reaction of VSCD with a His-tagged nanobody (i.e., a recombinant antibody fragment) raised against $T$. brucei and its loading with the drug nitrofurazone yields a delivery system that targets the surface of the parasite and that shows trypanocidal activity.

\section{Results and Discussion}

\section{Design and synthesis of a flexible carrier system}

Flexibility, defined as the capacity to: 1) transport a wide range of drugs (payload flexibility) and 2) form a stable link with any targeting element (target flexibility), was envisioned as a modular design that decouples therapeutic function (drug), carrier function (carrier element), and targeting function (biomolecule). The synthesis of such a flexible drug delivery system was articulated on a $\beta$-cyclodextrin $(\beta-C D)$ functionalized with a vinyl sulfone group (VS) to exploit the well-known capacity of CDs to form inclusion complexes with a wide variety of compounds, and the good reactivity, under mild conditions, of VS toward both amino and thiol groups naturally present in biomolecules. ${ }^{[17]}$ Recently, the functionalization of CDs with a zinc porphyrin has been reported as a general strategy to yield a multimodal system for combined therapy that takes advantage of the affinity of His and Trp residues for the metalloporphyrin center to couple CDs with a therapeutic protein. ${ }^{[18]}$
However, for drug delivery purposes the functionalization of CDs with VS is an advantageous strategy because it renders CDs into reactive species that yield a covalent bond (i.e., enhanced coupling stability) with any targeting element (enhanced target flexibility). In addition to the good reactivity toward biomolecules, the reaction is straightforward (it involves combining VSCD with the biomolecule in aqueous media), takes place under mild conditions compatible with the biological function of the targeting agent (aqueous buffer even at $\left.4{ }^{\circ} \mathrm{C}\right)$, and it does not yield by-products. ${ }^{[18 b, 19]}$

The synthesis of 6-deoxy-6-(2-hydroxyethyl) (vinylsulfonyl)methyl)amino- $\beta$-cyclodextrin (VSCD) was carried out in a twostep procedure consisting of: a) microwave-assisted reaction of 6-O-monotosyl-6- $\beta$-cyclodextrin ${ }^{[20]}$ with ethanolamine (1:20 stoichiometry) in DMF to yield mono-6-(2-hydroxyethyl)amino- $\beta$ cyclodextrin, and then $b$ ) introduction of VS by reaction with divinyl sulfone (DVS) (Scheme 1). The overall yield of the synthesis was $80 \%$. This strategy to obtain mono-6-(alkylamino)- $\beta$ cyclodextrin by displacement of the toluenesulfonyl group was

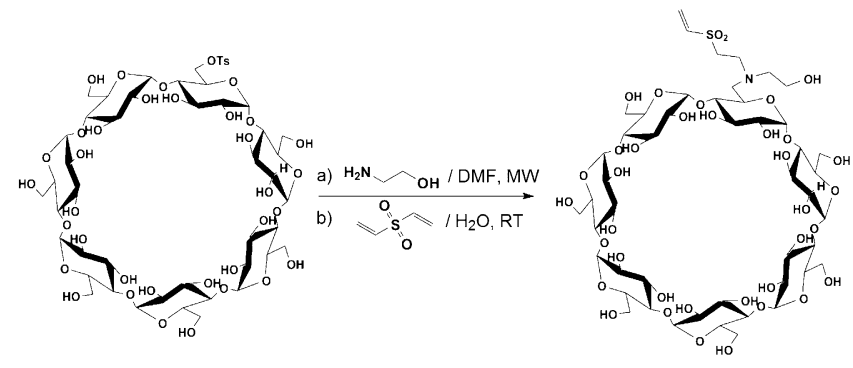

Scheme 1. Synthesis of 6-deoxy-6-(2-hydroxyethyl) (vinylsulfonyl)methyl)amino- $\beta$-cyclodextrin (VSCD).

recently reported by Puglisi et al., ${ }^{[21]}$ who used similar microwave conditions and a large molar excess of liquid amines (1:150), the amine also acting as solvent. Two side reactions may compromise the functionalization of mono-6-aminocyclodextrin with DVS: 1) dimerization by intermolecular reaction of both VS with two molecules of monoamino- $\beta$-cyclodextrin, and 2) intramolecular reaction of both VS with the amino group to yield a 1,1-dioxothiomorpholine derivative. The former was prevented by using DVS in 2:1 molar excess, and the latter by functionalizing the $\beta-C D$ as a secondary amine. Side reactions were minimized, as demonstrated by the fact that the yield of the reaction of DVS with mono-6-(2-hydroxyethyl)amino- $\beta$-cyclodextrin was $85.8 \%$.

\section{Selection of the in vitro model system to validate the proof of concept}

Trypanosomiasis was chosen as the model system to evaluate the potential of VSCD as a flexible carrier. There are three features that make trypanosomiasis attractive in this regard: 1) it is a relevant disease with more than 10 million people infected, 2) the current therapeutic arsenal has serious limitations and varied efficiency, as it relies on drugs that present an appreciable risk of toxicity, and drug delivery is a clear approach 
for optimization, ${ }^{[22]}$ and 3) trypanosomes are masters at eluding the host immune response. ${ }^{[23]}$ Our efforts were focused on Human African trypanosomiasis (HAT, or sleeping sickness) caused by $T$. brucei, because the mechanism of immune evasion relies on the antigenic variation of the variant surface glycoprotein coating (VSG) of the parasite, making targeting a particular challenge. ${ }^{[23]}$

Nitrofurazone (5-nitro-2-furfurylidenesemicarbazone; NF) was selected as payload. Although it is reported as an antiT. cruzi drug, ${ }^{[24]}$ several features attracted our attention: 1 ) it is active against $T$. brucei, ${ }^{[25]} 2$ ) it shows higher selectivity and lower toxicity than Nifurtimox, ${ }^{[26]}$ a classical anti-Chagas drug currently used in combination therapy against sleeping sickness, ${ }^{[27]}$ and 3 ) it is expected to be encapsulated by VSCD, as both hydroxymethylnitrofurazone and nitrofurazone have been included in $\beta-C D$ with similar $K_{\mathrm{a}}$ values, and the incorporation of hydroxymethylnitrofurazone in different substituted CDs has been reported. ${ }^{[28]}$

Selection of the targeting element was constrained by the fact that the antigenic variation of VSG makes T. brucei a difficult target for antibody-guided carriers, because conserved epitopes are located at the inner part of the coating, inaccessi-

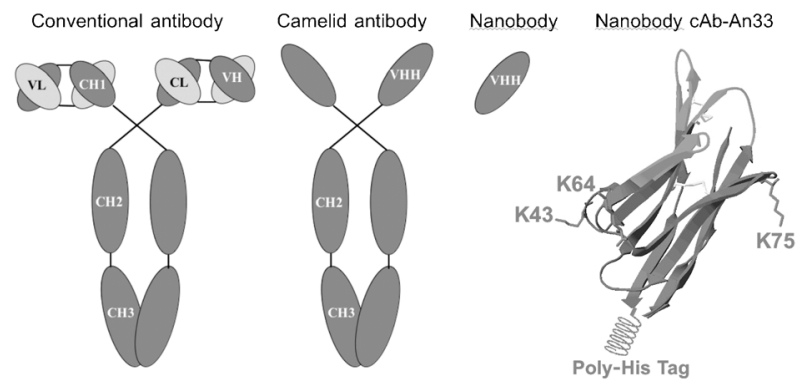

Figure 1. Antibodies and nanbodies. Conventional lgG antibodies consist of two heavy chains $(\mathrm{H})$ and two light chains $(\mathrm{L})$. The Camelidae family produces heavy-chain-only antibodies, the variable antigen binding domains (VHH) of which are termed nanobodies. Vinyl sulfone reactive residues of the poly-His-tagged CAb-An33 nanobody (at right) are labeled, and areas responsible for interaction with the antigen are shown in light grey, toward the top of the structure.

ble for standard antibodies (Figure 1). However, antibody fragments consisting of the variable antigen binding domain of the Camelid heavy-chain-only antibodies (i.e., nanobodies) have been demonstrated to target conserved cryptic epitopes. ${ }^{[29]}$ Additionally, nanobodies are well overexpressed in E. coli, are highly soluble in aqueous solution, and show such robust thermal and conformational stability that they have been reported to retain significant antigen binding activity even after incubation at $90^{\circ} \mathrm{C}$ for $2 \mathrm{~h}^{[30]}$ In particular, the cAbAn33 nanobody was chosen as a targeting element. It recognizes the carbohydrate moiety of VSG, and its structure has been solved at $1.6 \AA$ resolution, revealing a molecule $2.5 \mathrm{~nm}$ in diameter and $4.4 \mathrm{~nm}$ in length. ${ }^{[29,31]}$ Functionalization of the monoaminocyclodextrin was carried out with DVS, the shortest bis-vinyl sulfone, in order to minimize the size increase resulting from the coupling of VSCD to the nanobody, thereby pre- serving its capacity to interact with the conserved cryptic epitopes of T. brucei.

\section{Construction of the drug delivery system}

The modular design implies the assembly to the carrier element of the modules responsible for therapeutic function and for the targeting function in order to yield the drug delivery system. In principle, the order of integration is flexible and is determined by the features of both drug and targeting element. The presence of groups reactive toward VS in the drug means that the first reaction must involve coupling of VSCD to the targeting element. Conversely, when the conditions used to form the drug-CD inclusion complex may distort the functionality of the targeting element (i.e., solvents, temperature, $\mathrm{pH}$, etc.), inclusion should be carried out as the initial step.

\section{Coupling of VSCD to CAb-An33 nanobody}

The coupling of VSCD to proteins consists of a Michael addition that involves a nucleophilic attack at the side chains of Lys, Cys, and His residues by the VS, and the relative abundance of these residues can compromise the regioselectivity of the process. This issue may not be important for many of the '-omic' applications of vinyl sulfone chemistry. However, for coupling the VSCD to a guidance system, regioselectivity is crucial because if the linkage takes place at an unsuitable position, it may prevent interaction with the target. Analysis of the nanobody (Nb) structure (PDB code 1YC7) revealed that the reactive points are three Lys residues, as the four Cys residues are involved in two disulfide bonds (Figure 1). ${ }^{[1]}$ A closer study showed that only Lys75 is close enough to the antigen binding loops to disrupt the interaction with the target, whereas Lys64 and Lys43 are at opposite sides of the molecule. The reactivity of the Lys residues may be evaluated on the basis of the relationship between nucleophilic character and degree of protonation $\left(\mathrm{p} K_{\mathrm{a}}\right)$ because amine groups with lower $\mathrm{p} K_{\mathrm{a}}$ values are expected to be less protonated and behave as better nucleophiles, thus being more reactive. ${ }^{[32]}$ The prediction of $\mathrm{p} K_{\mathrm{a}}$ gave a value of $\sim 10$ for the three Lys residues, supporting a poor regioselectivity of the reaction between $\mathrm{Nb}$ and VSCD. To overcome such a limitation, the cAb-An33 nanobody was overexpressed as a poly-His tag fused protein. The poly-His tag is expected to act as a strong nucleophilic center, as the theoretical $\mathrm{p} K_{\mathrm{a}}$ for $\mathrm{His}$ residues is 6.5. ${ }^{[33]}$

To find the best reaction conditions, the poly-His-tagged CAb-An33 Nb was reacted with VS-functionalized rhodamine B (VSR). ${ }^{[17 \mathrm{~h}]}$ The reaction was carried out at two $\mathrm{pH}$ values and two Nb/VSR stoichiometries, and the functionality of the coupling was tested by incubation with parasites in vitro. Regardless of the stoichiometry, both $\mathrm{pH} 6$ and 7.5 yielded the labeling of $\mathrm{Nb}$ (Figure $2 \mathrm{a}$ ). However, a compromise between the extent of labeling and functionality was found, as strong fluorescence also correlates with multivalent labeling that may yield nonfunctional $\mathrm{Nb}$. It was found that $\mathrm{Nb}$ labeled with VSR at $\mathrm{pH} 7.5$ and a stoichiometry of 1:25 was fully functional and 
a) $\mathrm{pH} 6$
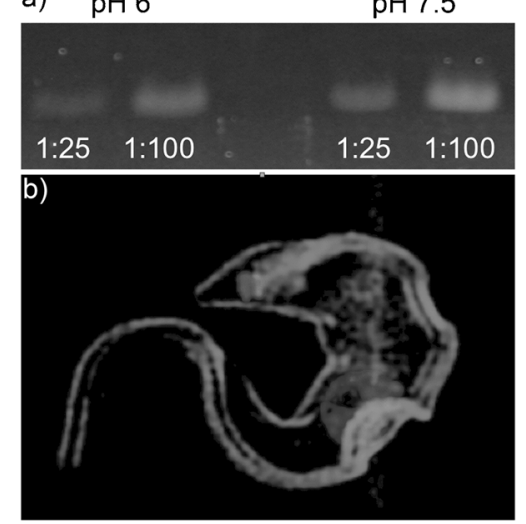

Figure 2. Reaction of the His-tagged cAb-An33 nanobody with vinyl sulfone rhodamine and interaction with T. brucei. a) SDS PAGE of the His-tagged nanobody having reacted with vinyl sulfone rhodamine $B$ at two $\mathrm{pH}$ values and two stoichiometries. b) Fluorescence resulting from the interaction of T. brucei with the His-tagged nanobody having reacted with vinyl sulfone rhodamine $\mathrm{B}$ at $\mathrm{pH} 7.5$ and at a stoichiometry of 1:25 (nucleus and kinetoplast are stained with DAPI).

targeted the entire surface of the parasite, validating the experimental approach (Figure $2 \mathrm{~b}$ ).

A finer-tuned approach was taken with VSCD. Different combinations of temperature, time, and reaction volume (i.e., final concentrations of the reacting species) were assayed, and the resulting products were analyzed by mass spectrometry (Supporting Information S1). The best conditions were found to be $1 \mathrm{nmol} \mathrm{Nb}$ and $25 \mathrm{nmol}$ VSCD in $60 \mu \mathrm{L}$, at $42^{\circ} \mathrm{C}$ in reaction for $24 \mathrm{~h}$ to yield Nb_VSCD with an overall labeling of $45 \%$ ( $29 \%$ single, $13 \%$ double, and $2 \%$ triple labeling). Harsher conditions caused the sample to precipitate.

To identify the residues that reacted with VS, two samples consisting of Nb_VSCD and Nb control were submitted to the Proteomic Facility (Universidad Autónoma de Barcelona, Spain) for digestion with trypsin and MALDI-ToF MS analysis. The spectra were searched for the signals of the peptides bearing Lys and His residues predicted by the in silico digestion of both $\mathrm{Nb}$ and Nb_VSCD samples (Supporting Information S2). As summarized in Table 1 and shown in Figure 3, the tryptic digest of the sample Nb_VSCD displays a clear signal that coincides with the $m / z$ value expected for the peptide resulting from the reaction between CDVS and the poly-His tail. Additionally, the MS data do not show the expected signal of the peptides containing $C D$ linked to Lys, except some traces for Lys64 (Supporting Information S3), confirming that VSCD is in-

Table 1. Overview of the signals searched in the MALDI-ToF spectra and summary of detection.

\begin{tabular}{|lcc|}
\hline Peptide bearing the residue target of VSCD & $\mathrm{m} / \mathrm{z}$ & Detected \\
\hline (R)QAPGK43CDE(R) & 2081.83 & No \\
(R)EWVSSISSPGTIYYQDSVK64CDGR(F) & 3655.56 & Trace \\
(R)DNAK75CDNTVYLQMNSLQR(E) & 3191.34 & No \\
(R)SIREYWGQGTQVTVSSHHHHHHCD(-) & 3916.34 & Yes \\
(R)EYWGQGTQVTVSSHHHHHHCD(-) & 3560.41 & Yes
\end{tabular}

(c) 2013 Wiley-VCH Verlag GmbH \&Co. KGaA, Weinheim a) SIREYWGQGTQVTVSSHннннн(CD) $\left(M_{r}=3916.3 \mathrm{Da}\right)$

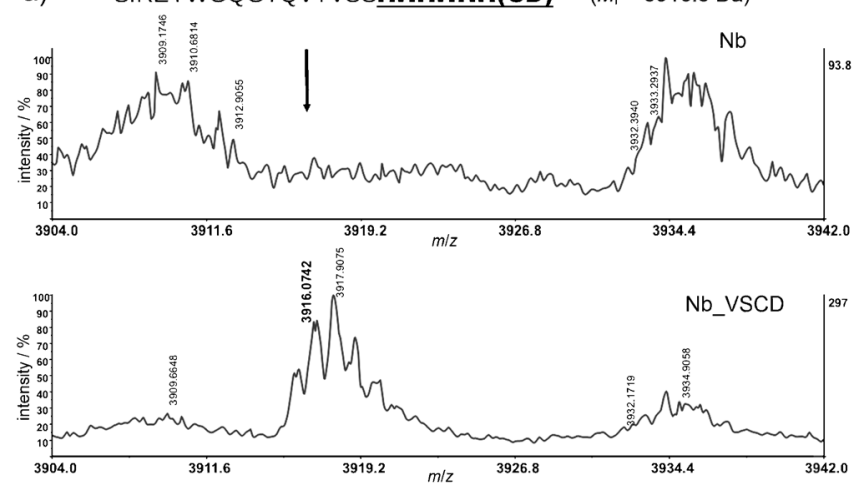

b) EYWGQGTQVTVSSHннннн(CD) $\left(M_{\mathrm{r}}=3560.4 \mathrm{Da}\right)$

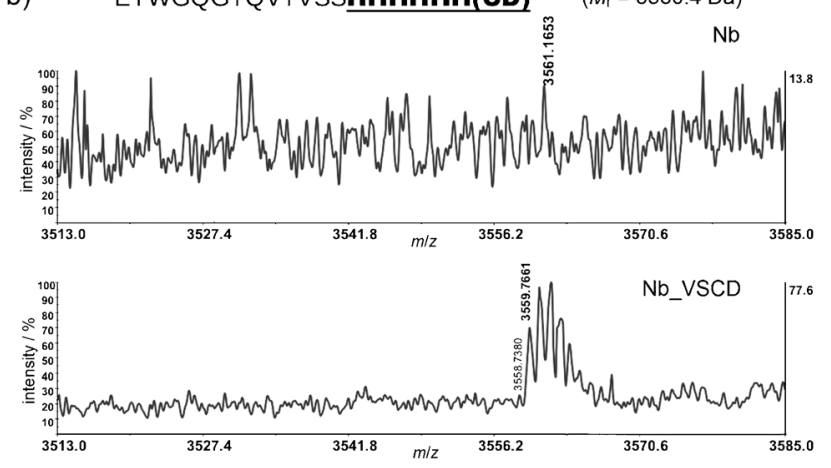

Figure 3. Detail of MALDI-ToF spectra of the two peptides bearing the polyHis tag derivatized with VSCD resulting from tryptic digestion of Nb_VSCD and $\mathrm{Nb}$ (control). The arrow indicates the expected $\mathrm{m} / \mathrm{z}$ value.

corporated mainly via reaction with the poly-His tail. This result is especially relevant, as His tags are widely used for the purification of recombinant proteins, and in the context of coupling via vinyl sulfone, it confers two additional advantages: 1) a degree of regioselectivity that restricts the coupling to other residues present in the biomolecules, and 2) reversibility if the construction of the fussed protein contains a protease cleavage site (typically for thrombin X) to remove the His tag.

\section{Inclusion of nitrofurazone}

The fact that the formation of inclusion complexes between hydroxymethylnitrofurazone and dimethyl- $\beta$-cyclodextrin have been reported ${ }_{1}^{[28]}$ and that docking experiments have shown the furan ring near the primary hydroxy group from $\mathrm{CD}^{[28 a]}$ led us to hypothesize that NF could form an inclusion complex with $\beta$-CD. Initial experiments aimed at forming inclusion complexes revealed the low solubility of NF in aqueous solutions as a significant obstacle that was overcome by including $1.5 \%$ $(v / v)$ DMSO in the medium. Although 1.5\% DMSO doubles the aqueous solubility of NF, T. brucei tolerance to DMSO is limited ${ }^{[34]}$ and the high solubility of NF in DMSO may prevent the formation of an inclusion complex with $C D$. It has been reported that the formation of the inclusion complex can be monitored by the absorbance of NF at $260 \mathrm{~nm} \cdot{ }^{[28 b]}$ This is not feasible for VSCD when coupled to proteins, as aromatic residues, particularly Phe, absorb in the $240-300 \mathrm{~nm}$ region, and disulfide bonds have an absorbance band close to $260 \mathrm{~nm}$. Howev- 
er, the UV/Vis spectrum of NF shows an additional strong absorbance band at $371 \mathrm{~nm}$ that is not affected by the presence of macromolecules (Supporting Information S4). The ratio $A_{371} /$ $A_{280}$ was used as an indicator of the formation of the inclusion complex. Thus, incubation of $5.4 \mathrm{nmol} \mathrm{Nb}$ _VSCD with $56.7 \mathrm{nmol} N \mathrm{NF}$ in $300 \mu \mathrm{L}$ phosphate-buffered saline (PBS) supplemented with $1.5 \%$ DMSO and exhaustive dialysis against PBS yielded a 1.4-fold increase in the $A_{371} / A_{280}$ ratio for the $N b_{-}$ VSCD + NF inclusion complex relative to that of Nb_VSCD, supporting the loading of Nb_VSCD with NF to yield Nb_VSCD + NF.

\section{Biological assays}

The therapeutic potential was evaluated by incubation of $20 \mu \mathrm{L}$ of either Nb_VSCD $+\mathrm{NF}$ (i.e., $15 \mu \mathrm{M}$ Nb_VSCD bearing $5 \mu \mathrm{M} \mathrm{NF}$ ) or control experiments with $80 \mu \mathrm{L}$ of a T. brucei culture in HMI-9 medium containing $2 \times 10^{5}$ parasites $\mathrm{mL}^{-1}$ for $5 \mathrm{~h}$ at $37{ }^{\circ} \mathrm{C}$ and $5 \% \mathrm{CO}_{2}$. The final concentration of DMSO in the biological assay was $0.3 \%(\mathrm{v} / \mathrm{v})$, which is well below the $0.42 \%$ threshold reported as safe for T. brucei, and the growth of the parasites was detected by Alamar Blue assay. ${ }^{[3]}$ This assay relies on the increase in fluorescence of a redox indicator in response to chemical reduction of the growth medium resulting from cell growth. Control experiments (PBS $+\mathrm{NF}$ and $\mathrm{Nb}+\mathrm{NF}$ ) yielded basically the same results as the positive control of parasite growth (PBS); only the culture in which the inclusion complex Nb_VSCD + NF was added yielded a decrease in fluorescence, revealing growth inhibition (Figure 4). The effectiveness of Nb_VSCD + NF was further confirmed by studying its effect on the growth kinetics of T. brucei. As shown in Figure 4, treatment for $4 \mathrm{~h}$ was sufficient to kill a culture of $2 \times$ $10^{5}$ parasites $\mathrm{mL}^{-1}$. These results demonstrate that both drug and carrier are necessary to inhibit growth, and that NF is not transported by the $\mathrm{Nb}$ unless it forms an inclusion complex with $C D$.

\section{Conclusions}

The functionalization of $\beta-C D$ with VS yields a drug carrier that combines the reactivity of VS toward biomolecules with the ability of $C D$ to form complexes with a wide range of drugs. This design decouples the carrier function from the targeting function to yield a versatile drug delivery system that may target various organs with different drugs upon coupling to the suitable targeting biomolecule and formation of the inclusion complex with the selected active principle. The reaction of VSCD with the biomolecule is straightforward and can be carried out without any special techniques. On the other hand the high reactivity of the His residue toward VS can be exploited to couple His-tagged guidance systems with a degree of regioselectivity that minimizes the disruptions of the interaction with the target molecule. As a proof of concept, the reaction of VSCD with $\mathrm{Nb}$ raised against $T$. brucei resulted in an effective drug delivery system that targets the surface of the parasite and shows trypanocidal activity when loaded with NF.
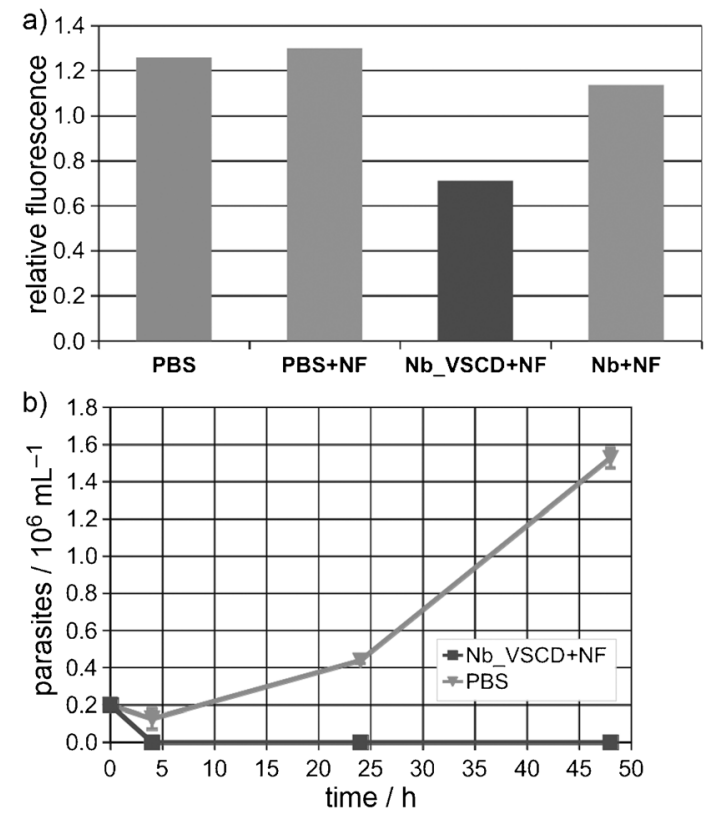

Figure 4. Trypanocidal activity of the transporter loaded with nitrofurazone. a) Alamar Blue assay of $T$. brucei after $7 \mathrm{~h}$ incubation with the inclusion complex (Nb_VSCD + NF), with negative controls in which Nb_CD was replaced by either PBS (PBS + NF) or Nb without CDVS (Nb + NF) or a positive control (PBS); values $<1$ denote an oxidized environment resulting from the absence of growth. b) Effect of Nb_VSCD + NF on the growth kinetics of T. brucei.

\section{Experimental Section}

General procedures: TLC was performed on Merck silica gel $60 \mathrm{~F}_{254}$ aluminum sheets. Flash column chromatography was performed on silica gel (Merck, $230-400$ mesh, ASTM). ${ }^{1} \mathrm{H}$ and ${ }^{13} \mathrm{C}$ NMR spectra were recorded at room temperature on a Varian Direct Drive $(400,500$, and $600 \mathrm{MHz})$ spectrometer. Chemical shifts $(\delta)$ are given in $\mathrm{ppm} ; J$ values are given in $\mathrm{Hz}$. MALDI-ToF mass spectra were recorded on an Autoflex Bruker spectrometer using HCCA and $\mathrm{Nal}$ as matrices. Optical rotations were recorded on a PerkinElmer 341 polarimeter at room temperature. Vinyl sulfone derivatized rhodamine (VSR) was obtained as described elsewhere. ${ }^{[17 \mathrm{~h}]} \mathrm{Ni}-$ trofurazone (5-nitro-2-furfurylidenesemicarbazone) was purchased from Alfa Aesar.

Synthesis of 6-deoxy-6-(2-hydroxyethyl) (vinylsulfonyl)methyl)amino- $\beta$-cyclodextrin (VSCD): Ethanolamine $(470 \mu \mathrm{L}, 7.75 \mathrm{mmol})$ was added to a solution of 6-toluenesulfonyl- $\beta$-cyclodextrin $(500 \mathrm{mg}, 0.38 \mathrm{mmol})$ in DMF $(10 \mathrm{~mL})$. The reaction mixture was irradiated at $500 \mathrm{~W}$ and $80^{\circ} \mathrm{C}$ in a Milestone Star Microwave Labstation for $1 \mathrm{~h}$. Acetone was added $(100 \mathrm{~mL})$, and the precipitate was filtered in vacuo and washed with acetone $(20 \mathrm{~mL})$ and $\mathrm{Et}_{2} \mathrm{O}$ $(20 \mathrm{~mL})$ to yield 6-deoxy-6-hydroxylethylamino- $\beta$-cyclodextrin as a solid that was dried in vacuo at $50^{\circ} \mathrm{C}$ for one day $(410 \mathrm{mg}$, $90 \%$ ). ${ }^{[20]}$ Divinylsulfone $(26 \mu \mathrm{L}, 0.25 \mathrm{mmol}$ ) was added to a solution of 6-deoxy-6-hydroxylethylamino- $\beta$-cyclodextrin (150 mg, $0.13 \mathrm{mmol})$ in $\mathrm{H}_{2} \mathrm{O}(15 \mathrm{~mL})$. The reaction mixture was kept at room temperature for $1 \mathrm{~h}$. The solvent was evaporated in vacuo (to $\sim 8 \mathrm{~mL})$. Acetone was added $(50 \mathrm{~mL})$, and the precipitate was filtered in vacuo and washed with acetone $(10 \mathrm{~mL})$ and $\mathrm{Et}_{2} \mathrm{O}(10 \mathrm{~mL})$. The solid was dried in vacuo at $50^{\circ} \mathrm{C}$ for one day $(140 \mathrm{mg}, 85 \%)$ : $\mathrm{mp}>270^{\circ} \mathrm{C}(\mathrm{dec}) ;[\alpha]_{\mathrm{D}}=+102\left(c=1, \mathrm{H}_{2} \mathrm{O}\right) ;{ }^{1} \mathrm{H}$ NMR (selected signals, $\left[\mathrm{D}_{6}\right.$ ]DMSO, $\left.300 \mathrm{MHz}\right): \delta=6.90(\mathrm{dd}, J=16.6,9.9 \mathrm{~Hz}, 1 \mathrm{H}), 6.20$ $(\mathrm{d}, J=9.7 \mathrm{~Hz}, 1 \mathrm{H}, \mathrm{HC}=c i s), 6.17 \mathrm{ppm}(\mathrm{d}, J=16.6 \mathrm{~Hz}, 1 \mathrm{H}, \mathrm{HC}=$ 
trans); ${ }^{13} \mathrm{C}$ NMR ([D $]$ DMSO, $\left.75 \mathrm{MHz}\right): \delta=138.0,129.8,102.7,82.2$, 73.8, 73.1, 72.7, 60.6, 59.8, 57.0, 55.5, 51.0, 48.6 ppm; HRMS (MALDI-ToF): $[\mathrm{M}+\mathrm{H}]^{+}$calcd for $\mathrm{C}_{48} \mathrm{H}_{83} \mathrm{O}_{37} \mathrm{NS}: 1296.428$, found: 1296.452.

Overexpression and purification of nanobody CAb-An33: Production of the poly-His-tagged cAb-An33 nanobody ( $\mathrm{Nb}$ ) was performed as described elsewhere. ${ }^{[31]}$

Coupling of $\mathrm{Nb}$ to VSCD to yield Nb_VSCD: A final reaction volume of $60 \mu \mathrm{L}$ containing $1 \mathrm{nmol} \mathrm{Nb}$ and $25 \mathrm{nmol}$ VSCD in $100 \mathrm{~mm}$ HEPES pH 7.5 was incubated at $42^{\circ} \mathrm{C}$ for $24 \mathrm{~h}$. The sample was thoroughly dialyzed against PBS to remove excess vinyl sulfone derivatized reagent and was used without further purification.

Mass spectrometry analysis of Nb_VSCD: MS was carried out at the Proteomic Facility at Universidad Autónoma de Barcelona (Spain). For localization of the VSCD in Nb, an aliquot of a solution of either $\mathrm{Nb}$ or Nb_VSCC was reduced with DTT, derivatized with iodoacetamide, and digested with trypsin $\left(37^{\circ} \mathrm{C}, 16 \mathrm{~h}\right)$. The resulting peptides were analyzed by MALDI-ToF MS (ABI-Sciex 4800) in linear and reflection mode to cover a range between 700 and $6000 \mathrm{Da}$. The mass spectra were interpreted manually on the basis of the in silico digestion of the sequence. The molecular masses of the VSCD-derivatized peptides were calculated by adding the molecular mass of VSCD to the peptide with His or Lys.

Inclusion of nitrofurazone into Nb VSCD: Nb VS $(5.4 \mathrm{nmol})$ in $300 \mu \mathrm{L}$ PBS was incubated with a solution of nitrofurazone $(4.5 \mu \mathrm{L}$, $12.6 \mathrm{~mm}$ in DMSO) at room temperature overnight. Excess nitrofurazone was removed by dialysis against PBS $(3 \times 100 \mathrm{~mL})$. Inclusion was monitored by UV/Vis spectroscopy as the ratio $A_{371} / A_{280}$.

\section{Biological assays}

Trypanosoma brucei brucei AnTat 1.1 bloodstream parasites were cultured in HMI-9 medium supplemented with $20 \%$ tetracyclinefree fetal bovine serum (FBS) at $37^{\circ} \mathrm{C}$ in an atmosphere of $5 \% \mathrm{CO}_{2}$ in air.

Fluorescence microscopy: Freshly isolated trypanosomes were diluted in PBS to a final concentration of $0.5-0.7 \times 10^{6}$ parasites $\mathrm{mL}^{-1}$ and fixed in $4 \%(\mathrm{v} / \mathrm{v})$ paraformaldehyde. After washing with PBS $(1 \mathrm{~mL})$, the suspension was incubated at $37^{\circ} \mathrm{C}$ for $1 \mathrm{~h}$ with a $0.25 \mathrm{mg} \mathrm{mL}^{-1}$ nanobody labeled with vinyl sulfone derivatized rhodamine $B$ solution. It was then washed with PBS and to fixed onto poly-L-lysine-coated slides for $30 \mathrm{~min}$ at $37^{\circ} \mathrm{C}$ in a wet camera. Finally, slides were washed twice with PBS and once with water, mounted with Vectashield containing DAPI (Vector Laboratories) and visualized by immunofluorescence microscopy (Olympus IX81). Deconvolution of 3D images was performed with Huygens Essential software (version 2.9; Scientific Volume Imaging) and the restored image data set was visualized and analyzed with EZ-C1 3.30 FreeViewer software.

Alamar Blue assays: Exponentially growing parasites were harvested and adjusted with buffer to a cell density of $2 \times 10^{5}$ trypanosomes per $\mathrm{mL}$ to ensure that cells were in logarithmic growth phase during the entire experiment. Trypanosome culture $(80 \mu \mathrm{L})$ was added to each well of flat-bottom 96-well plate containing $20 \mu \mathrm{L}$ test sample. Cultured plates were incubated at $37^{\circ} \mathrm{C}$ in an atmosphere of $5 \% \mathrm{CO}_{2}$ for $5 \mathrm{~h}$ before the addition of $20 \mu \mathrm{L}$ of the colorimetric viability indicator resarzurin sodium salt at $0.5 \mathrm{~mm}$. After further incubation for $2 \mathrm{~h}$, the reaction was stopped by the addition of a $3 \%$ SDS solution $(50 \mu \mathrm{L})$. The plates were read on a Tecan Infinite F200 reader (Tecan Austria GmbH, Austria) using an excitation wavelength of $535 \mathrm{~nm}$ and an emission wavelength of $590 \mathrm{~nm}$.

Trypanolysis assays: A culture $(400 \mu \mathrm{L})$ of $2 \times 10^{5}$ parasites $\mathrm{mL}^{-1}$ in HMl-9 medium supplemented with $20 \%$ tetracycline-free FBS was incubated at $37^{\circ} \mathrm{C}$ and $5 \% \mathrm{CO}_{2}$ with $100 \mu \mathrm{L}$ test samples. After 4 , 24 , and $48 \mathrm{~h}$ incubation, the parasites were counted with a Neubauer chamber.

\section{Abbreviations}

$\mathrm{CD}$, cyclodextrin; $\mathrm{Nb}$, poly-His-tagged CAb-An33 nanobody; NF, nitrofurazone; DVS, divinylsulfone; VS, vinyl sulfone group; VSCD, vinyl sulfone functionalized $\beta$-cyclodextrin; VSR, vinyl sulfone functionalized rhodamine $\mathrm{B}$; Nb_VSCD, Nb coupled to VSCD; Nb_VSR, $\mathrm{Nb}$ coupled to VSCR.

\section{Acknowledgements}

The authors and indebted to Mr. José Maceira for skillful technical assistance. This work was funded by grants CTQ2011-29299C02-01.A to F.S.-G. and SAF-2011-30528 to J.A.G.S. from the Ministerio de Economía y Competitividad (Spain) and by the European Union (NANOTRYP, FP7-HEALTH-2007-B-2.3.4-1:223048) to J.A.G.S. and S.M.

Keywords: bioconjugation $\cdot$ cyclodextrins $\cdot$ drug delivery vinyl sulfones

[1] a) K. Strebhardt, A. Ullrich, Nat. Rev. Cancer 2008, 8, 473-480; b) Y. H. Bae, K. Park, J. Controlled Release 2011, 153, 198-205; c) S. Sultana, M. R. Khan, M. Kumar, S. Kumar, M. Ali, J. Drug Targeting 2013, 21, 107 25

[2] M. Yokoyama, J. Artif. Organs 2005, 8, 77-84.

[3] H. Maeda, J. Wu, T. Sawa, Y. Matsumara, K. Hori, J. Controlled Release $2000,65,271-284$

[4] a) C. Moorthi, R. Manavalan, K. Kathiresan, J. Pharm. Pharm. Sci. 2011, 14, 67-77; b) P. Sapra, T. M. Allen, Cancer Res. 2002, 62, 7190-7194 c) A. M. Koch, F. Reynolds, R. Weissleder, L. Josephson, ChemBioChem 2005, 6, 337-345; d) E. Mahon, A. Salvati, F. B. Bombelli, I. Lynch, K. A Dawson, J. Controlled Release 2012, 161, 164-74; e) N. K. Mehra, V. Mishra, N. K. Jain, Ther. Delivery 2013, 4, 369-94.

[5] D. C. Litzinger, A. M. J. Buiting, N. van Rooijen, L. Huang, Biochim. Biophys Acta 1994, 1190, 99-107.

[6] Y. S. Rhee, H. M. Mansour, Int. J. Nanotechnol. 2011, 8, 84-114; b) R. Haag, F. Kratz, Angew. Chem. 2006, 118, 1218-1237; Angew. Chem. Int Ed. 2006, 45, 1198-1215.

[7] a) D. M. Goldenberg, R. M. Sharkey, Oncogene 2007, 26, 3734-3744; b) R. M. Sharkey, D. M. Goldenberg, Ca-Cancer J. Clin. 2006, 56, 226 243 ; c) I. Pastan, R. Hassan, D. J. Fitzgerald, R. J. Kreitman, Nat. Rev. Cancer 2006, 6, 559-565; d) O. Nelson, MMG 445 Basic Biotechnology eJournal 2008, 4, 3-12, http://ejournal.vudat.msu.edu (Michigan State University); e) X.-Q. Qiu, H. Wang, B. Cai, L.-L. Wang, S.-T. Yue, Nat. Biotechnol. 2007, 25, 921 -929; f) C. Enever, T. Batuwangala, C. Plummer, A. Sepp, Curr. Opin. Biotechnol. 2009, 20, 405-411; g) B. A. Teicher, R. V. J. Chari, Clin. Cancer Res. 2011, 17, 6389-6397; h) D. Lipovsek, Protein Eng. Des. Sel. 2011, 24, 3-9; i) P. Martin-Killias, N. Stefan, S. Rothschild, A. Pluckthun, U. Zangemeister-Wittke, Clin. Cancer Res. 2011, 17, 100-110; j) R. R. White, B. A. Sullenger, C. P. Rusconi, J. Clin. Invest. 2000, 106, 929-934; k) O. C. Farokhzad, J. Cheng, B. A. Teply, I. Sherifi, S. Jon, P. W. Kantoff, J. P. Richie, R. Langer, Proc. Natl. Acad. Sci. USA 2006, 103, 6315-6320; I) V. C. Ozalp, F. Eyidogan, H. A. Oktem, Pharmaceuticals 2011, 4, 1137-1157; m) X. Ni, M. Castanares, A. Mukherjee, S. E. Lupold, Curr. Med. Chem. 2001, 18, 4206-4214; n) Y. Zhang, H. Hong, W. Cai, Curr. Med. Chem. 2011, 18, 4185-4194. 
[8] a) X. B. Zhao, R. J. Lee, Adv. Drug Delivery Rev. 2004, 56, 1193-1204 b) H. Elnakat, M. Ratnam, Front. Biosci. 2006, 11, 506-519; c) G. Destito, R. Yeh, C. S. Rae, M. B. Fin, M. Manchester, Chem. Biol. 2007, 14, 1152 1162; d) C. Zhang, L. Zhao, Y. Dong, X. Zhang, J. Lin, Z. Chen, Eur. J. Pharm. Biopharm. 2010, 76, 10-16.

[9] a) M. Singh, Curr. Pharm. Des. 1999, 5, 443-451; b) H. linuma, K. Maruyama, K. Okinaga, K. Sasaki, T. Sekine, O. Ishida, N. Ogiwarea, K. Hohkura, Y. Yonemura, Int. J. Cancer 2002, 99, 130-137; c) T. R. Daniels, T. Delgado, G. Helguera, M. L. Penichet, Clin. Immunol. 2006, 121, 159 176; d) T. R. Daniels, E. Bernabeu, J. A. Rodriguez, S. Patel, M. Kozman, D. A. Chiapetta, E. Holler, J. Y. Ljubimova, G. Helguera, M. L. Penichet, Biochim. Biophys. Acta Gen. Subj. 2012, 1820, $291-317$.

[10] T. Wang, T. Weil, Chem. Today 2009, 27, 6-10.

[11] a) R. Weissleder, K. Kelly, E. Y. Sun, T. Shtatland, L. Josephson, Nat. Biotechnol. 2005, 23, 1418-1423; b) M. Shi, J. Lu, M. S. Shoichet, J. Mater. Chem. 2009, 19, 5485-5498.

[12] L. Nobs, F. Buchegger, R. Gurny, E. Allemann, Int. J. Pharm. 2003, 250, $327-337$.

[13] S. Balthasar, K. Michaelis, N. Dinauer, H. von Briesen, J. Kreuter, K. Langer, Biomaterials 2005, 26, 2723-2732.

[14] G. Saito, J. A. Swanson, K. D. Lee, Adv. Drug Delivery Rev. 2003, 55, 199215.

[15] a) J. J. Yin, Z.-W. Zhou, S.-F. Zhou, Drug. Deliv. And Transl. Res. 2013, 3, 364-375; b) J. J. Hin, S. Sharma, S. P. Shumyak, Z.-X. Wang, Z.-W. Zhou, Y. Zhang, P. Guo, C.-Z. Li, J. R. Kanwar, T. Yang, S. S. Mohapatra, W. Liu, W. Duan, J.-C. Wang, Q. Li, X. Zhang, J. Tan, L. Jia, M. Q. Wei, X. Li, S.-F. Zhou, PLoS One 2013, 8, 362289; c) A. Okamatsu, K. Motoyama, R. Onodera, T. Higashi, T. Koshigoe, Y. Shimada, K. Hattori, T. Takeuchi, H. Arima, Bioconjugate Chem. 2013, 24, 724-733; d) H. Arima, A. Yoshimatsu, H. Ikeda, A. Ohyama, K. Motoyama, T. Higashi, A. Tsuchiya, T. Niidome, Y. Katayama, K. Hattori, T. Takeuchi, Mol. Pharm. 2012, 9, 2591 2604; e) H. Zhang, Z. Cai, Y. Sun, F. Yu, Y. Chen, B. Sun, J. Biomed. Mater Res. 2012, 100A, $2441-2449$; f) P. Caliceti, S. Salmaso, A. Semenzato, T. Carofiglio, R. Fornasier, M. Fermeglia, M. Ferrone, S. Pricl, Bioconjugate Chem. 2003, 14, 899-908; g) Q. Zhou, X. Guo, T. Chen, Z. Zhang, S. Shaou, C. Luo, J. Li, S. Zhuo, J. Phys. Chem. B 2011, 115, 12662-12679, h) S. Salmaso, S. Bersani, A. Semenzato, P. Caliceti, J. Drug Targeting 2007, 15, 379-390; i) Y. Yang, Y. M. Zhang, Y. Chen, D. Zhao, J. T. Chen, Y. Liu, Chemistry 2012, 18, 4208-4215; j) A. Miao, S. Li, S. Han, Z. Wang, Y. Wu, G. Nie, J. Nanopart. Res. 2012, 14, 1-14; k) G. J. L. Bernardes, R. Kikkeri, M. Magliano, P. Laurino, M. Collot, S. Y. Hong, B. Lepenies, P. H. Seeberger, Org. Biomol. Chem. 2010, 8, 4987-4996; I) Y. Oda, H. Yanagisawa, M. Maruyama, K. Hattori, T. Yamanoi, Bioorg. Med. Chem. 2008, 16, 8830-8840; m) K. Hattori, A. Kenmoku, T. Mizuguchi, D. Ikeda, M. Mizuno, T. Inazu, J. Inclusion Phenom. Macrocyclic Chem. 2006, 56, 916 ; n) J. M. Benito, M. Gomez-Garcia, C. Ortiz-Mellet, I. Baussanne, J. Defaye, J. M. G. Fernandez, J. Am. Chem. Soc. 2004, 126, 10355-10363.

[16] G. Tiwari, R. Tiwari, A. K. Rai, J. Pharm. BioAllied Sci. 2010, 2, 72-79.

[17] a) R. Arun, K. C. K. Ashok, V. V. N. S. S. Sravanthi, Sci. Pharm. 2008, 76, 567-598; b) J. Porath, R. Raxen, Methods Enzymol. 1976, 44, 19-45; c) N. S. Simpkins, Tetrahedron 1990, 46, 6951-6984; d) G. Houen, O. M Jensen, Immunol. Methods 1995, 181, 187-200; e) M. S. Masri, M. Friedman, J. Protein Chem. 1998, 7, 49-54; f) F. J. López-Jaramillo, F. PerezBanderas, F. Hernandez-Mateo, F. Santoyo-Gonzalez, Acta Crystallogr. Sect. F 2005, 61, 435-438; g) D. C. Meadows, J. Gervay-Hague, Med. Res. Rev. 2006, 26, 793-814; h) J. Morales-Sanfrutos, J. Lopez-Jaramillo, M. Ortega-Muñoz, A. Megia-Fernandez, F. Perez-Balderas, F. HernandezMateo, F. Santoyo-Gonzalez, Org. Biomol. Chem. 2010, 8, 667-675.
[18] a) Z. Kejik, T. Briza, J. Kralova, P. Pouckova, A. Kral, P. Martasek, V. Kral, Bioorg. Med. Chem. Lett. 2011, 21, 5514-5520; b) Z. Kejik, T. Briza, P. Pouckova, J. Kralova, V. Kral, P. Martasek, J. Controlled Release 2008, 132, e27; c) J. Kralova, Z. Kejik, T. Briza, P. Pouckova, A. Kral, P. Martasek, V. Kral, J. Med. Chem. 2010, 53, 128-138.

[19] a) F. J. Lopez-Jaramillo, M. Ortega-Muñoz, A. Megía-Fernandez, F. Hernandez-Mateo, F. Santoyo-Gonzalez, Bioconjugate Chem. 2012, 23, 846 855 ; b) M. Ortega-Muñoz, J. Morales-Sanfrutos, A. Megía-Fernandez, J. Lopez-Jaramillo, H. Hernandez-Mateo, F. Santoyo-Gonzalez, J. Mater. Chem. 2010, 20, 7189-7196.

[20] N. Zhong, H.-S. Byun, R. Bittman, Tetrahedron Lett. 1998, 39, 2919-2910.

[21] A. Puglisi, J. Spencer, J. Clarke, J. Milton, J. Inclusion Phenom. Macrocyclic Chem. 2012, 73, 475-478.

[22] O. Kayser, O. Olbrich, S. L. Croft, A. F. Kiderlen, Parasitol. Res. 2003, 90, S63-S70.

[23] A. Oladiran, M. Belosevic, J. Parasitol. 2012, 98, 284-292.

[24] a) H. Cerecetto, M. Gonzalez, Pharmaceuticals 2010, 3, 810-838; b) J. A. Urbina, Acta Trop. 2010, 115, 55-68.

[25] G. Chauviere, B. Bauteille, B. Enanga, C. de Albuquerque, S. L. Croft, M. Dumas, J. Perie, J. Med. Chem. 2003, 46, 427-440.

[26] A. Gerpe, G. Alvarez, D. Benitez, L. Boiani, M. Quiroga, P. Hernandez, M. Sortino, S. Zacchino, M. González, H. Cerecetto, Bioorg. Med. Chem. 2009, 17, 7500-7509.

[27] Human African trypanosomiasis (sleeping sickness), Fact Sheet No. 259, World Health Organization, 2012: http://www.who.int/mediacentre/factsheets/fs259/en/ (accessed November 28, 2013).

[28] a) R. Grillo, N. F. S. de Melo, D. R. de Araujo, G. H. G. Trossini, E. F. F. da Cunha, T. de Castro Ramalho, L. F. Fraceto, J. Inclusion Phenom. Macrocyclic Chem. 2010, 66, 417-421; b) N. F. S. de Melo, R. Grillo, A. H. Rosa, L. F. Fraceto, J. Pharm. Biomed. Anal. 2008, 47, 865-869; c) R. Grillo, N. F. S. De Melo, L. F. Fraceto, C. L. Brito, G. H. G. Trossini, C. M. S. Menezes, E. I. Ferreria, Quim. Nova 2008, 31, 290-295; d) R. Grillo, N. F. S. de Melo, C. M. Moraes, R. de Lima, C. M. S. Menezes, E. I. Ferreria, A. H. Rosa, L. F. Fraceto, J. Pharm. Biomed. Anal. 2008, 47, 295-302; e) R. Grillo, N. Ferreria, F. S. Melo, C. M. Moraes, A. H. Rosa, J. A. F. Roveda, C. M. S. Menezes, E. I. Ferreira, L. F. Fraceto, J. Biol. Phys. 2007, 33, 445453.

[29] B. Stijlemans, K. Conrath, V. Cortez-Retamozo, H. Van Xong, L. Wyns, P. Senter, H. Revets, P. De Baetselier, S. Muyldermans, S. Magez, J. Biol. Chem. 2004, 279, 1256-1261.

[30] R. H. J. van der Linden, L. G. J. Frenken, B. de Geus, M. M. Harmsen, R. C. Ruuls, W. Stok, L. de Ron, S. Wilson, P. Davis, C. T. Verrips, Biochim. Biophys. Acta Protein Stuct. Mol. Enzymol. 1999, 1431, 37-46.

[31] K. Conrath, C. Vincke, B. Stijlemans, J. Schymkowitz, K. Decanniere, L. Wyns, S. Muyldermans, R. Loris, J. Mol. Biol. 2005, 350, 112-125.

[32] F. J. Lopez-Jaramillo, F. Hernandez-Mateo, F. Santoyo-Gonzalez, Vinyl Sulfone: A Multi-Purpose Function in Proteomics, Integrative Proteomics (Ed. Hon-Chiu Eastwood Leung), InTech, Rijeka, 2012, pp. $301-326$.

[33] J. A. Traverso, F. J. Lopez-Jaramillo, A. J. Serrato, M. Ortega-Muñoz, D. Aguado-Lleda, M. Sahrawy, M. F. Santoyo-González, J. L. Neira, A. Chueca, J. Plant Physiol. 2010, 167, 423-429.

[34] M. L. Syked, V. M. Avery, Am. J. Trop. Med. Hyg. 2009, 81, 665-674.

Received: September 26, 2013

Revised: November 20, 2013

Published online on $\square$ II, 0000 


\section{FULL PAPERS}

T. del Castillo, J. Marales-Sanfrutos,

F. Santoyo-González, S. Magez,

F. J. Lopez-Jaramillo, * J. A. Garcia-Salcedo

$\square-\square$

Monovinyl Sulfone $\beta$-Cyclodextrin. A Flexible Drug Carrier System

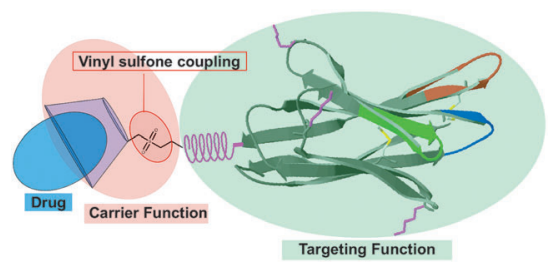

HAT on the hit list: Monovinyl sulfone $\beta$-cyclodextrin is a flexible drug carrier that can target various organs with different drugs when coupled to a suitable targeting biomolecule and upon formation of an inclusion complex with the selected active principle. Its coupling to a nanobody raised against $T$. brucei and loading with nitrofurazone yields a system that targets the surface of the parasite and shows trypanocidal activity. 